\title{
Pulsations of A stars
}

\author{
Luis A. Balona \\ South African Astronomical Observatory, \\ P.O. Box 9, Observatory 7935, Cape Town, South Africa \\ email: lab@saao.ac.za
}

\begin{abstract}
A review of recent work on pulsating A stars is presented. The types of pulsating stars are the roAp, $\delta$ Scuti, $\lambda$ Bootis, Am stars, pre-Main Sequence pulsating A stars and $\alpha$ Cygni stars (pulsating A supergiants). Population II pulsating A stars such as SX Phe (blue stragglers) and RR Lyraes are also discussed. The emphasis is on the physics that can be derived from the study of stellar pulsations and suggestions for further progress.
\end{abstract}

Keywords. Stars: oscillations, stars: chemically peculiar

\section{Introduction}

The A-type stars present possibly the most interesting and challenging problems in stellar pulsation. They lie on the hot side of the Classical Instability Strip and include all evolutionary states from pre-Main Sequence to supergiants and also Population II pulsators such as RR Lyraes. Pulsating chemically peculiar and magnetic stars such as the roAp stars offer additional problems and opportunities.

In this paper I will discuss what can be learned from the pulsations in A-type stars, presenting the most recent results from the literature and examining what we have learned, or may learn from such studies.

\section{The roAp stars}

The roAp stars are the most complex pulsating stars. The observed pulsations are strongly influenced by the magnetic field and are affected by horizontal spatial chemical composition variations and vertical stratification. Furthermore, the pulsation frequencies are near the critical acoustic limit with wavelengths comparable with the pressure scale height in the atmosphere. As a result, it is not sufficient to use a simple one-layer approximation to model the line profile variations.

In the atmosphere the pulsations are, in fact, magneto-hydrodynamic waves with both longitudinal and transverse components. The eigenfunctions do not resemble a simple combination of normal p-mode eigenfunctions. Despite the very high pulsation frequency, when in normal stars one may expect the displacement to be almost entirely vertical, the horizontal displacement component is probably significant because of coupling with the magnetic field. In addition, the form of the eigenfunction probably varies across the photosphere due to the differing geometry of the magnetic field. As a result, I believe we are a long way from understanding and modeling the line profile variations. Nevertheless, these complexities present unique opportunities for understanding the interaction between pulsations and magnetic fields.

The first step in any asteroseismological investigation is to determine the mode of pulsation. Owing to the complex nature of the eigenfunctions, well-known photometric and spectroscopic methods applicable to other pulsating stars fail for roAp stars. In addition, the pulsation frequencies are modified quite significantly by the interaction with 
the magnetic field. As a result, the roAp stars are not suitable objects for asteroseismology on a detailed scale.

Recent high-resolution spectroscopic observations appear to show that the pulsations are apparently nonaxisymmetric (Kochukhov \& Ryabchikova, 2001ab). Furthermore, the phase of the pulsations appears to vary from ion to ion, indicative of the small scale length and, possibly, a running wave component. These observations remain to be explained and, on first sight, are at odds with the very successful and generally accepted oblique pulsator model. It should be noted, however, that Shibahashi (2005) has proposed that the apparent nonaxisymmetric line profiles could possibly be understood as due to a shock wave in an axisymmetric mode. This is an interesting idea which deserves further study.

Bigot \& Dziembowski (2002) introduced two main improvements in understanding the pulsations in magnetic stars. They treated the effect of the magnetic field with a nonpertubative theory and included the effects of the centrifugal distortion of the star. They found that rotation plays an important role, even in the slow rotators. As a result, the magnetic and pulsation axes are no longer aligned.

The mechanism responsible for exciting the oscillations in roAp stars is still unknown. Balmforth et al. (2000) suggested that the high magnetic field intensity at the poles will suppress convection. Freed of this damping, the star may pulsate in this region driven by the $\kappa$ mechanism acting in the hydrogen ionization zone. Cunha (2002) estimated the theoretical edges of the instability strip using such models. Comparison with observations show that most roAp stars fall well within this strip, though mostly on the cooler side. Cunha suggests that the Ap stars within the instability strip which do not pulsate (the "noAp" stars) may, in fact, be pulsating with periods which are longer than expected. They may be undetected because of the observational technique normally used to discover roAp stars.

Cunha et al. (2003) attempted to constrain theoretical models which characterise the pulsations in HR 1217. They find that the interior chemical composition is more important than the modeling of convection and that lower abundances of heavy elements and larger abundance of helium lead to a closer match to the data.

Very recently, there has been several papers which examine the apparent variations in the magnetic field over a pulsational cycle. Leone \& Kurtz (2003) found evidence of such an effect in the Nd III lines of $\gamma$ Equ. Savanov et al. (2003) offer confirmation, but further observations by Kochukhov et al. (2004) do not show this effect. Even if such an effect is securely established, it will be difficult to model the line profiles for reasons already mentioned. As a result, it may not be possible to understand the physics involved in the process and, therefore, to make any firm deductions.

Further progress in our understanding of the roAp stars is likely to come from time resolved high-dispersion spectroscopy and from space observations. We need to understand the cause of the apparently nonaxisymmetric modes giving rise to the line profile variations and the variation of pulsation phase in the atmosphere. Space observations should uncover many new very low amplitude modes which could be very important for a full understanding of the interaction between pulsation and magnetic fields. An interesting possibility would be the detection of a chromosphere or corona around roAp stars. Because the pulsation frequency is so close to the acoustic limit, it is possible that the running wave component may deposit sufficient energy in the upper atmosphere to produce the necessary elevated temperature.

On the theoretical side, the main unsolved problem is the unknown excitation mechanism. The proposal by Balmforth et al. (2000) is promising, but requires further study. 


\section{3. $\delta$ Scuti stars}

In many ways $\delta$ Scuti stars are probably the best candidates for asteroseismology, especially the hotter stars for which the effects of convection can be ignored. Unlike roAp stars, they have "simple" atmospheres and the pulsation frequencies are considerably higher than the rotational frequencies so that the eigenfunctions of the pressure modes are well approximated by simple spherical harmonics. The usefulness of these stars arises from the large number of modes that have been observed in a few cases. Breger et al. (1998) detected at least 24 significant frequencies of low degree in the A5 star FG Vir. Recent observations have more than doubled this number.

A large number of observed frequencies is an important requirement for asteroseismology as it constrains the solution. Of equal importance are the mode identifications and a model which is a good approximation to the star. Mantegazza \& Poretti (2002) have recently obtained line profile observations of FG Vir which are in general agreement with the photometric mode identifications, but suffer from an uncertainty of \pm 1 in the degree, $\ell$. This uncertainty is, unfortunately, typical of line profile analyses, independently of the $S / N$ ratio of the data, and negates much of the effort that is required to obtain these data. They do, however, provide valuable information on the inclination angle which in turn is necessary to apply rotational corrections to the frequencies. It has been shown that even a relatively small rotational velocity has a great impact on the expected frequencies leading to serious errors in matching the observed frequencies with models. Interactions in the eigenfunctions of closely spaced modes distort the mode identifications, especially in moderately and rapidly rotating stars.

Although the pulsation modes of most frequencies in FG Vir appear to be rather well identified from photometric techniques, a complete and unique astroseismic solution has still to be found, in spite of intensive efforts (Breger et al. 1999). One is beginning to suspect that there is something wrong with models of the "simple" envelopes of A-type stars. Templeton et al. (2001) find that changes to the stellar mass, the chemical composition and the convective core overshooting length change the observed pulsation spectrum significantly. It, therefore, seems that there are more unobservables than previously supposed. This stresses the importance of detecting as many frequencies as possible.

Most observations thus far have concentrated on modes of low degree which are, in fact, the most useful from an asteroseismological point of view. However, modes of high degree have been detected in some stars (e.g., $\tau$ Peg, Kennelly et al. 1998). These modes can only be detected if the star has a moderate to high projected rotational velocity. It is possible that many modes of high degree may become observable with MOST, although establishing the spherical harmonic degree may be an impossible task.

It seems to me that the modeling of pulsations in $\delta$ Scuti stars is well behind the observations. It would be interesting to explore the effects of changing the models in various ways to determine which physical processes lead to a better match with the data.

\section{4. $\lambda$ Bootis stars}

The $\lambda$ Boo stars are Population I late B- to early F-type stars with moderate to extreme surface underabundances of most Fe-peak elements and solar abundances of the lighter elements. Paunzen et al. (2002a) find that these stars comprise a homogeneous group containing stars at all phases of the Main Sequence evolution. This suggests that the process for depleting the Fe-peak elements works continuously during the Main Sequence phase. 
In an investigation of the pulsations of $\lambda$ Boo stars, Paunzen et al. (2002b) found that at least $70 \%$ of all $\lambda$ Boo stars within the Classical Instability Strip pulsate and they do so with high overtone modes. These results indicate that there is probably no clear difference between $\lambda$ Boo stars and $\delta$ Sct stars as far as pulsational properties are concerned. Thus it seems reasonable to suppose that the peculiar abundances are confined to the surface and do not significantly affect the pulsations. The challenge remains, of course, as to the mechanism whereby the chemical peculiarities come about.

Paunzen et al. (2003) observed several $\lambda$ Boo stars in the near infrared with the object of detecting circumstellar material. They found evidence for an infrared excess in a quarter of the sample of stars. Gray \& Corbally (2002) continued a program to search for CP A stars, particularly $\lambda$ Boo stars, in open clusters. It now appears that no $\lambda$ Boo stars occur in clusters. It would seem that some factor external to the star and related to membership in open clusters prevents the operation of the $\lambda$ Boo phenomenon. They suggest that this may be a result of the photoevaporation of circumstellar material around these stars by UV radiation from massive cluster stars.

Paunzen (2005) has suggested that the peculiar abundances in $\lambda$ Boo stars originate by accretion when the star passes through an interstellar dust cloud. There seems to be convincing evidence that this idea is correct.

\section{Pulsations in Am stars}

The classical Am stars are A-type stars whose spectra are characterized by an underabundance of $\mathrm{Ca}$ (and/or Sc) coupled with an overabundance of the Fe group and heavier elements with respect to normal stars of the same colour. Originally it was thought that Am stars do not pulsate because diffusion drains helium from the ionization zone which is responsible for driving the pulsations. This is consistent with the idea that diffusion is the mechanism for the line-strength anomalies of the Am and Ap stars.

Observations of several Am stars show that this simple model is not valid; pulsation and metallicism have been found to co-exit in classical Am stars, though pulsating Am stars are rare. Pulsations in evolved Am stars and marginal Am stars lying near the red edge of the instability strip can be understood within the context of diffusion theory in terms of driving by residual He II and the $\mathrm{H}$ ionization zone. Most of the observed pulsating Am stars seem to be of this class (e.g., HD 98851 and HD 102480, Joshi et al. 2003, HD 13079, Martinez et al. 1999). However, Am stars near the ZAMS are not expected to pulsate (Turcotte et al. 2000).

The complexities regarding the pulsations in Am stars was nicely summarized by Kurtz (2000). In a nutshell, the slowly rotating A-type stars appear as chemically peculiar because of diffusion and generally do not pulsate because the $\kappa$ mechanism can no longer operate in the depleted He II ionization zone. However, evolved Am stars (the $\rho$ Pup or $\delta$ Del stars) pulsate due to an evolutionary replenishment of some helium in the He II ionization zone. In marginal Am stars the He II ionization zone is not fully depleted, so low-amplitude pulsations can occur. In other words, diffusion in those Am stars which pulsate is assumed not to have completely drained helium from the He II ionization zone so that the $\kappa$ mechanism can still operate, leading to low-amplitude pulsations.

To test these ideas, one needs a clearer understanding of the incidence of low amplitude pulsations in the A-star Main Sequence band. The role of photometric space observations of Am stars and apparently non-pulsating Main Sequence A-type stars is, therefore, of great importance for further progress. 


\section{Pulsations in pre-Main Sequence A-type stars}

Intermediate mass pre-Main Sequence stars are expected to cross the instability strip on their way to the Main Sequence. The Herbig Ae stars are thought to be such objects as they are found in star forming regions and show the characteristics of infalling material. Although they normally exhibit significant irregular light variations, pulsation in these stars is not difficult to find because of the periodic nature and the short period. Catala (2003) has presented a nice review of pulsations in these stars.

Breger (1972) was the first to identify two pre-Main Sequence pulsators in the open cluster NGC 2264. The bright Herbig Ae star HR 5999 was found to be pulsating by Kurtz \& Marang (1995) and Kurtz \& Catala (2001). Strangely, the pulsations in this star do not seem to be detectable in high-dispersion spectroscopic observations (Balona, unpublished).

Marconi et al. (2000) discovered short-period variability in two Herbig Ae stars V351 Ori and HD 35929. Marconi et al. (2001) found that V351 Ori pulsates with at least four frequencies. A detailed photometric and spectroscopic analysis of V351 Orionis by Balona et al. (2002) shows that two frequencies are definitely present in the light curve and a further frequency is visible in the radial velocity variations. Furthermore, modes of high degree $(\ell \approx 8)$ are sometimes visible.

Kurtz \& Muller (2001) discovered pulsations in the light curve of the Herbig Ae star HD 142666, but could not detect pulsations in another Herbig Ae star, HD 142527, lying within the instability strip.

HD 104237 (A4V) is of particular interest. Donati et al. (1997) detected an unambiguous magnetic field (the first time detection of a magnetic field in a Herbig Ae star) and small amplitude radial velocity variations with a $37-$ min period. Recent results (Böhm et al. 2004) show that the star is a spectroscopic binary and pulsates with at least five periods. This star is a multiple object, there being at least 5 components (Feigelson et al. 2003) with four low-mass PMS companions.

Ripepi et al. (2002) obtained photometric time series observations of pre-Main Sequence stars in IC 348. They found one star, H254, which has a single mode with frequency 7.406 cycles $\mathrm{d}^{-1}$. Ripepi et al. (2003) present multisite observations of V353 Ori in which 5 frequencies are detected. Pinheiro et al. (2003) detected two periods in V346 Ori.

Perhaps the most remarkable discovery in this regard is that of pulsations in the archetypal young A-type star $\beta$ Pictoris. Koen (2003) discovered three frequencies in the light variations of $\beta$ Pic. Subsequent high-resolution spectroscopic observations (Koen et al. 2003) revealed at least 18 frequencies, mostly of high degree $(4<\ell<10)$. Comparisons with models suggest that the observed frequencies are compatible with those expected for a $\delta$ Scuti star with about the same temperature and luminosity.

The hotter A-type stars are not expected to be chromospherically active. The detection of strong emission lines of O VI and C III with FUSE in $\beta$ Pic (Bouret et al. 2002) came as a complete surprise. The data are compatible with the presence of an active chromosphere in $\beta$ Pic. Whether the presence of a chromosphere may be a result of energy transport by pulsation is an open question.

\section{Pulsation in A supergiants}

The variations in A supergiants are extremely complex and involve timescales from hours to years. Variations can occur in either the photosphere or the extended envelope that is associated with mass loss. The early work by Abt (1957), who observed the radial 
velocity of several A- and F-type supergiants for nearly every night for 30 consecutive nights, still provides an important benchmark. All the stars proved to be variable with characteristic periods in the range 4 - 30 days.

The most extensively studied A supergiant is $\alpha$ Cyg. Lucy (1976) performed a periodogram analysis of 144 velocities which suggest that the variability is due to the simultaneous excitation of many discrete pulsation modes with periods ranging from 6.9 to $100.8 \mathrm{~d}$. The expected radial fundamental mode for $\alpha$ Cyg is about $14 \mathrm{~d}$. Since that time, the only spectroscopic work of note on these stars is that of Kaufer et al. (1997). They obtained time series spectra of A-type and late B-type supergiants. They find complex cyclical variations of the radial velocities with typical velocity dispersion of about $3 \mathrm{~km} \mathrm{~s}^{-1}$. Multiple periods both longer and shorter than the radial fundamental mode are present. Examination of the line profile variations show prograde traveling features. These could be g-modes of low degree $(\ell<5)$.

Adelman \& Albayrak (1997) examined the Hipparcos database for variability of A0-A5 supergiants in the Bright Star Catalogue. They find that the amplitude increases with luminosity, but that no definite periods can be determined from the data other than those for the three A supergiants examined by the Hipparcos team. The periods given for these three stars are $6.36 \mathrm{~d}$ (HR 618), $1.33 \mathrm{~d}$ (HR 4169) and $2.36 \mathrm{~d}$ (HR 6825), all considerably shorter than the radial fundamental mode.

Because of the long periods and the large amount of data required to extract a periodic signal from the irregular variations which characterise these stars, very little work has been done. The most promising approach is a dedicated program of observations on an automatic photometric telescope.

\section{SX Phe stars and pulsating blue stragglers}

Blue stragglers are stars (mostly found in globular clusters) which are probably formed by stellar mergers from short-period binaries or collisions. These stars fall in a region of the colour-magnitude diagram consistent with having masses greater than the current cluster turnoff. The field star, SX Phe, is sometimes identified as a possible blue straggler. A remarkably high fraction of blue stragglers exhibit variations associated with binary interactions and pulsation. Some of them show two radial modes, which allows the mass to be constrained.

The most comprehensive investigation of pulsating blue stragglers is that of Gilliland et al. (1998). Observations of six SX Phe variables in the core of 47 Tuc were obtained using HST. Two of these stars show pulsations in the fundamental and first-overtone mode, two others in the forth and fifth radial overtone while the remaining two variables are multiperiodic variables. From these data they were able to estimate the masses which are well above the the turnoff mass in 47 Tuc and are consistent with the merger scenario.

\section{RR Lyrae stars and the Blazhko effect}

The nature of the Blazhko effect in RR Lyrae stars remains an unsolved problem. The effect is the slow periodic, or quasi-periodic, modulation of the amplitude and shape of the light curve. One possibility is that it is due to the modulation of the pulsations during a magnetic cycle. However, Chadid et al. (2004) found no evidence for the presence of a magnetic field in RR Lyrae, the brightest Blazhko star.

Other evidence which seems to exclude models involving rotational effects is that the Blazhko period does not remain constant. Jurcsik et al. (2002) find that the pulsation 
period of XZ Dra exhibits cyclic, but not strictly regular variations with $\mathrm{a} \approx 7200 \mathrm{~d}$ period and that the Blazhko period seems to follow the observed period changes of the radial fundamental mode. LaCluyze et al. (2004) found the Blazhko period in XZ Cyg is anti-correlated with the primary period of XZ Cyg. These are important results because they exclude any explanation which requires that the Blazhko period be exactly equal or directly proportional to the rotation period of the star.

Recent attempts to explain the Blazhko effect have focused on resonance models, in which there is a nonlinear resonance between the dominant radial mode and a nonradial mode, or the oblique magnetic rotator. Nowakowski (2002) and Nowakowski \& Dziembowski (2001) find that significant amplitude and phase modulation of the light curve might occur from a pulsation in which the pair of modes $\ell=1, m= \pm 1$ are excited. The Blazhko period is then determined by the rotation frequency weighted with the BruntVaisala frequency in the deepest part of the radiative envelope of the star. It is not clear whether this model can explain the correlation or anti-correlation of the Blazhko period with the fundamental radial mode.

The detection and identification of nonradial modes in RR Lyrae stars would be an important achievement for our understanding of the Blazhko effect. To this end, Kolenberg (2002) and Chadid et al. (1999) performed detailed frequency analysis of line profile variations in RR Lyr and obtained strong evidence for the presence of nonradial modes. Time resolved high dispersion spectra of RR Lyr stars seem to be a promising new tool for understanding the Blazhko effect.

\section{Conclusions}

The roAp stars present us with a large number of unsolved problems, of which the enigmatic line profile variations and phase shifts are just two examples. Progress is hampered because we do not yet fully understand the eigenfunction. Further observations of time-resolved line profile variations will undoubtedly assist in resolving this very difficult problem.

It seems to me that observations of pulsations in $\delta$ Scuti stars are well ahead of theory. We need to understand why it is so difficult to match the observed frequencies with those from models. Definite progress has recently been made in our understanding of the $\lambda$ Boo stars (Paunzen 2005). It is likely that high precision photometry from space will enable very low amplitude Am stars to be detected and thus confirm our understanding of how pulsations are driven in these stars.

Detection of pulsations in pre-Main Sequence stars is necessary to map out the preMS instability strip, although we need to refine estimates of temperature and luminosity. Pulsations offer a possible mechanism of energy deposition in the upper atmosphere and may lead to the creation of a chromosphere. The evidence for chromospheres on preMain Sequence Vega-type stars and Herbig Ae stars is convincing and opens new lines of research.

There has been very little progress on observations on SX Phe stars, blue stragglers and supergiants. To some extent this can be attributed to the faintness of the former and the long-term observational effort in the latter.

Some progress is at last being made on the long-standing puzzle of the Blazhko effect in RR Lyrae stars. It is now clear that the Blazhko period is coupled in some way with the pulsation period. The challenge of detecting the nonradial modes responsible for the coupling is probably best addressed by high resolution spectroscopy. 


\section{References}

Abt, H.A. 1957, ApJ 126, 138

Adelman, S.J. \& Albayrak, B. 1997, IBVS 4541

Balmforth, N.J., Cunha, M.S., Dolez, N., Gough, D.O. \& Vauclair, S. 2000, MNRAS 323, 362

Balona, L.A., Koen, C. \& van Wyk, F. 2002, MNRAS 333, 923

Bigot, L. \& Dziembowski, W.A. 2002, A\& A 391235

Böhm, T., Catala, C., Balona, L.A. \& Carter, B. 2004, A\&SA, in press.

Bouret, J.-C., Deleuil, M., Lanz, T., Roberge, A., Lecavelier des Etangs, A. \& Vidal-Madjar, A. 2002, A\&̈A 390, 1049

Breger, M. 1972, ApJ 171, 539

Breger, M., Zima, W., Handler, G., et al. 1998, A\&SA 331, 271

Breger, M., Pamyatnykh, A.A., Pikall, H. \& Garrido R. 1999, A\&̛A 341, 151

Catala, C. 2003, ApSS 284, 53

Chadid, M., Kolenberg, K., Aerts, C. \& Gillet, D. 1999, A\&A 352, 201

Chadid, M., Wade, G.A., Shorlin, S.L.S. \& Landsteet, J.D. 2004, A\&A 413, 1087

Cunha, M.S. 2002, MNRAS 333, 47

Cunha, M.S., Fernandes, J.M.M.B. \& Monteiro M.J.P.F.G. 2003, MNRAS 343, 831

Donati, J-F., Semel, M., Carter, B.D., Rees, D.E. \& Cameron, A.C. 1997, MNRAS 291, 658

Feigelson, E.D., Lawson, W.A. \& Garmire, G.P. 2003, ApJ 599, 1207

Gilliland, R.L., Bono, G., Edmonds, P.D., Caputo, F., Cassisi, S., Petro, L.D., Saha, A. \& Shara, M.M. 1998, ApJ 507, 818

Gray, R.O. \& Corbally, C.J. 2002, AJ 124, 989

Joshi, S., Girish, V., Sagar, R., Kurtz, D.W., Martinez, P., Kumar, B., Seetha, S., Ashoka, B.N. \& Zhou, A. 2003, MNRAS 344, 431

Jurcsik, J., Benko, J.M. \& Szeidl, B. 2002, A\&\&A 396, 539

Kaufer, A., Stahl, O., Wolf, B., Fullerton, A.W., Gaeng, T., Gummersbach, C.A., Jankovics, I., Kovacs, J., Mandel, H., Peitz, J., Rivinius, T. \& Szeifert T. 1997, A\&3A 320, 273

Kennelly, E.J., Brown, T.M., Kotak, R., Sigut, T.A.A., Horner, S.D., Korzennik, S.G., Nisenson, P., Noyes, R.W., Walker, A. \& Yang S. 1998, ApJ 495, 440

Kochukhov, O. \& Ryabchikova, T. 2001a, A\&A A 374, 615

Kochukhov, O. \& Ryabchikova, T. 2001b, A\&A 377, L22

Kochukhov, O., Ryabchikova, T. \& Piskunov, N. 2004, A\& A 415, 13

Koen, C. 2003, MNRAS 341, 1385

Koen, C., Balona, L.A., Khadaroo, K., Lane, I., Prinsloo, A., Smith, B. \& Laney, C.D. 2003, MNRAS 344, 1250

Kolenberg, K. 2002, A spectroscopic study of the Blazhko effect in RR Lyrae, Ph.D. Thesis, University of Leuven

Kurtz, D.W. \& Marang F. 1995, MNRAS 276, 191

Kurtz, D.W. 2000, in Delta Scuti and Related Stars, ed. M. Breger \& M. H. Montgomery (San Francisco: ASP Vol 210), 287

Kurtz, D.W. \& Catala C. 2001, A\& A 369, 981

Kurtz, D.W. \& Muller, M. 2001, MNRAS 325, 1341

LaCluyze, A., Smith, H.A., Gill, E.-M., Hedden, A., Kinemuchi, K., Rosas, A.M., Pritzl, B.J., Sharpee, B., Wilkinson, C., Robinson, K.W., Baldwin, M.E. \& Samolyk, G. 2004, astro$\mathrm{ph} / 0401314$

Leone, F. \& Kurtz, D.W. 2003, A\&A 407, 67

Lucy, L.B. 1976, ApJ 206, 499

Mantegazza, L. \& Poretti, E. 2002, A\&SA 396, 911

Marconi, M., Ripepi, V., Alcala, E., Covino, E., Palla, F., \& Terranegra L. 2000, A\& A 355, L35.

Marconi, M., Ripepi, V., Bernabei, S., Palla, F., Alcala, E, Covino, E. \& Terranegra, L. 2001, $A \mathscr{E} A$ 372, L21.

Martinez, P., Kurtz, D.W., Ashoka, B.N., Chaubey, U.S., Gupta, S.K., Leone, F., Catanzaro, G., Sagar, R., Raj, E., Seetha, S. \& Kasturirangan, K. 1999, MNRAS 309, 871

Nowakowski, R.M. \& Dziembowski, W.A. 2001, Acta Astron. 51, 5 
Nowakowski, R. 2002, in Radial and Noradial Pulsations as Probes of Stellar Physics, ed. C. Aerts, T.R. Bedding \& J. Christensen-Dalsgaard (San Francisco: ASP), 408

Paunzen, E., Iliev, I. Kh., Kamp, I. \& Barzova, I.S. 2002a, MNRAS 336, 1030.

Paunzen, E., Handler, G., Weiss, W.W., Nesvacil, N., Hempel, A., Romero-Colmenero, E., Vuthela, F.F., Reegen, P., Shobbrook, R.R., Kilkenny, D. 2002b, A\&A 392, 515

Paunzen, E., Kamp, I., Weiss, W.W. \& Weisemeyer, H. 2003, A $\mathscr{\ddots} A$ 404, 579

Paunzen, E. 2005, These Proceedings, 443

Pinheiro, F.J.G., Folha, D.F.M., Marconi, M., Ripepi, V., Palla, F., Monteiro, M.J.P.F.G. \& Bernabei, S. 2003, A $\mathscr{E} A$ 399, 271

Ripepi, V., Palla, F., Marconi, M., Bernabei, S., Arellano Ferro, A., Terranegra, L. \& Alcala, J.M. 2002, A\&SA 391, 587

Ripepi, V., Marconi, M., Bernabei, S., et al. 2003, A\&SA 408, 1047

Savanov, I., Musaev, F.A. \& Bondar, A.V. 2003, IBVS 5468

Shibahashi, H. 2005, These Proceedings, GP17

Simon, T., Ayres, T.R., Redfield, S. \& Linsky, J.L. 2002, ApJ 579, 800

Templeton, M., Basu, S. \& Demarque, P. 2001, ApJ 563, 999

Turcotte, S., Richer, J., Michaud, G. \& Christensen-Dalsgaard, J. 2000, A\&A 360, 603

\section{Discussion}

SAIO: I would like to mention that $\delta$ Scuti type pulsations in roAp stars can be suppressed by a magnetic field larger than about $1 \mathrm{kG}$, according to a nonadiabatic analysis.

BALONA: That would certainly help us to understand why we do not find $\delta$ Sct pulsations in roAp stars.

BREGER: You are absolutely correct in concluding that refined models are most important for asteroseismology of $\delta$ Scuti stars. May I add that, in addition, observations supporting these refinements need to be made whenever possible.

BALONA: Yes, I agree. I believe we need to change the current models of A stars used in the pulsational analysis.

ShiBAhAshi: In the case of $\beta$ Pic, are the low-degree modes found photometrically detected by spectroscopy?

BALONA: The answer is no, but I believe this is due to the low amplitudes of the modes. The low-degree modes cause long-scale line profile variations which are more difficult to detect.

SkodA: Could the high order line profile variations in $\beta$ Pic be explained by periodic circumstellar obscuration rather than pulsation?

BALONA: The line profiles described here are detected in all photospheric lines and cannot be due to absorption by the circumstellar material.

KUPKA: I would like to point out that Simon et al. (2002) have presented FUSE observations of a number of A-type stars. They find signatures of chromospheric emission for stars cooler than $8300 \mathrm{~K}$, but not for hotter stars. 
BALONA: $\beta$ Pictoris, with $T_{\text {eff }}=8200 \mathrm{~K}$, is in the hot end of the temperature range of the chromospherically active stars detected by Simon et al. (2002). My point is that the detection of a chromosphere in these stars is difficult to understand because we do not know how the very thin convective region can support magnetic activity. Bouret et al. (2002) show that their model of a thin region heated up to a few $10^{5} \mathrm{~K}$ located close to the photosphere reproduces the observations in $\beta$ Pic remarkably well. The challenge is to understand how such a chromosphere-transition region can be formed in these stars. 